\title{
Characteristics of Takayasu Arteritis Patients with Severe Ischemic Events
}

\author{
Richard Ying Yu, Roaa AlSolimani, Nader Khalidi, Christian Pagnoux, and Lillian Barra, \\ for the Canadian Vasculitis Network (CanVasc)
}

\begin{abstract}
Objective. Takayasu arteritis (TA) is a rare large-vessel vasculitis that puts patients at high risk of developing severe ischemic events (SIE). Outcomes for TA patients with SIE are poorly understood. We aim to describe the characteristics of TA patients experiencing SIE.

Methods. All TA patients with at least 1 followup visit seen between 1988 and 2015 were included from 3 academic centers in Ontario, Canada. Diagnosis was based on American College of Rheumatology criteria, physician opinion, and vascular imaging. SIE were defined as cerebrovascular accident (CVA), acute coronary syndrome (ACS), ischemic cardiomyopathy, ischemic blindness, and/or ischemic bowel or limb requiring surgery.

Results. Of the 52 patients with TA included in the study, 51 (98\%) were female and $22(42 \%)$ were of European descent. The mean age was 31 (SD 12) at the time of diagnosis and the followup time was 6 years (SD 5). Fifteen (29\%) experienced an SIE: 5 CVA, 5 ACS, 1 ischemic cardiomyopathy, and 4 limb ischemia. Thirteen out of 15 SIE (87\%) occurred at or before diagnosis. Patients with SIE were more likely than those without SIE to be started on corticosteroids combined with immunosuppressants $(p=0.04)$ and antiplatelet agents $(p=0.004)$. Outcomes including disease activity and damage scores were similar between patients with and without SIE.

Conclusion. SIE are common in patients with TA and occur early in the disease. With aggressive treatment, patients with SIE had a favorable prognosis. (First Release April 15 2020; J Rheumatol 2020;47:1224-8; doi:10.3899/jrheum.190407)
\end{abstract}

Key Indexing Terms:

TAKAYASU ARTERITIS ISCHEMIA CARDIOVASCULAR LARGE-VESSEL VASCULITIS

Takayasu arteritis (TA) is a rare form of granulomatous vasculitis, characterized by inflammation of the aorta and its major branches, and is found most commonly in young Asian females $^{1,2}$. Diagnosis remains challenging for several reasons, including its rarity, lack of disease awareness, heterogeneous

From the Schulich School of Medicine and Dentistry, Western University, London, Ontario; Department of Medicine, Division of Rheumatology, St. Joseph's Health Care London, University of Western Ontario, London, Ontario; Division of Rheumatology, St. Joseph's Healthcare, McMaster University, Hamilton, Ontario; Vasculitis Clinic, Division of Rheumatology, Mount Sinai Hospital, University of Toronto, Toronto, Ontario, Canada.

R.Y. Yu was funded by the Canadian Rheumatology Association (CRA)-Pfizer Summer Research Studentship.

R.Y.Yu, BSc, Schulich School of Medicine and Dentistry, Western University; R. AlSolimani, MD, Department of Medicine, Division of Rheumatology, St. Joseph's Health Care London, University of Western Ontario, and Consultant of Internal Medicine, Rheumatologist, King Abdulaziz University, Jeddah, Saudi Arabia; N. Khalidi, MD, Division of Rheumatology, St. Joseph's Healthcare, McMaster University; C. Pagnoux, MD, MPH, Vasculitis Clinic, Division of Rheumatology, Mount Sinai Hospital, University of Toronto; L. Barra, MD, MPH, Schulich School of Medicine and Dentistry, Western University, and Department of Medicine, Division of Rheumatology, St. Joseph's Health Care London, University of Western Ontario.

Address correspondence to Dr. L. Barra, Division of Rheumatology, Department of Medicine, St. Joseph's Health Care London, University of Western Ontario, 268 Grosvenor St., Room D2-160, London, Ontario N6A4V2, Canada.E-mail: lillian.barra@sjhc.london.on.ca

Accepted for publication September 30, 2019. disease presentations, and early nonspecific symptoms ${ }^{2,3}$. A major consequence of delayed TA diagnosis is stenosis and occlusion of vessels leading to end-organ ischemia ${ }^{2,3}$.

A recent metaanalysis demonstrated that patients with TA have high rates of developing severe ischemic events (SIE) ${ }^{4}$. The most commonly reported forms of SIE are strokes, transient ischemic attacks (TIA) ${ }^{4,5}$, and acute coronary syndromes $(\mathrm{ACS})^{4,6}$. Other manifestations of SIE, including ischemic induced blindness ${ }^{7}$, ischemic limb requiring surgical intervention ${ }^{8}$, and ischemic gut ${ }^{9}$, are not as consistently reported in the literature ${ }^{4}$. In addition, there is a lack of studies describing characteristics, treatment, and outcomes of patients with SIE over time.

In this study, we investigated patients with TA from a Canadian cohort with long followup, to describe the clinical characteristics of those patients with SIE and their management.

\section{MATERIALS AND METHODS}

The patients included in our study were seen at 3 Canadian academic centers: St. Joseph's Health Care in London, Mount Sinai Hospital in Toronto, and St. Joseph's Healthcare in Hamilton (all in Ontario) ${ }^{10}$. All patients with TA seen at these centers between 1988 and 2015 were included in the study if they had available and complete data at diagnosis, at least 1 followup visit, and if written informed consent was obtained. Diagnosis was based on American College of Rheumatology (ACR) classification criteria ${ }^{11}$ and/ or physician opinion based on clinical presentation and vascular imaging.

Personal non-commercial use only. The Journal of Rheumatology Copyright () 2020. All rights reserved. 
The study was approved by the research ethics boards of each site: REB1000012364, 102078, and 15-034.

Patient data were collected at diagnosis, and when available at 6 months, 12 months, every relapse, and last followup using standardized forms. Information collected consisted of demographics, medical history, and comorbidities [including traditional cardiovascular (CV) risk factors: diabetes, hypertension, dyslipidemia, and obesity], clinical manifestations, laboratory and imaging investigations, current treatments or interventions, and the following composite measure: the Indian Takayasu Activity Score (ITAS2010) $^{12}$. ITAS was completed retrospectively. To confirm diagnosis, all patients underwent vascular imaging by computed tomography angiography (CTA), magnetic resonance angiography (MRA), and/or conventional digital-subtraction angiography. For followup, the usual practice at our centers is to perform vascular imaging for a suspected TA flare that cannot be confirmed by clinical examination or to identify complications (aneurysm or critical ischemia). Positron emission tomography (PET) and MRA are not readily available at our centers; CTA for followup is avoided to reduce radiation exposure.

SIE were defined as cerebrovascular accident (CVA; stroke or TIA), ACS [ST elevation myocardial infarction (MI; STEMI) or non-STEMI (NSTEMI)], ischemic cardiomyopathy, ischemia-induced blindness, or limb or bowel ischemia requiring surgical intervention. Stroke was defined by a neurologist as an ischemic or hemorrhagic event causing acute focal neurologic signs and/or symptoms and confirmed by magnetic resonance imaging (MRI). TIA was defined as a temporary neurologic ischemic event with reversible focal neurological signs and/or symptoms. ACS and ischemic cardiomyopathy were diagnosed by a cardiologist based on clinical symptoms and confirmed by electrocardiography, echocardiography, laboratory investigations, and/or coronary angiography. Limb ischemia was determined based on clinical findings of reduced vascular flow (i.e., decreased pulses, cyanosis, cutaneous ulcers, gangrene) that required surgery (including amputation, vascular bypass, arthroplasty, and stent placement). Ischemia-induced blindness was defined by an ophthalmologist on examination of the retina, and bowel ischemia requiring surgical intervention was determined by pathology of the surgical specimen. The following ischemic manifestations were not considered severe and were excluded: Raynaud phenomenon, ischemic neuropathy, syncope due to decreased cerebral blood flow, renal ischemia not requiring dialysis, cutaneous ulcerations, digital cyanosis/periungal infarcts, claudication symptoms, or bowel ischemia not requiring surgical intervention.

Active TA was defined by the treating physician as new or worsening symptoms attributable to TA or ongoing symptoms with elevations in acute-phase reactants (APR) and/or evidence of disease activity by imaging. Remission was the absence of symptoms, elevations in APR, or imaging findings of active TA disease. Relapse was defined by the recurrence of TA symptoms, new symptoms, or progression of vascular lesions on imaging, with or without an elevation in APR, and leading to a change in treatment in patients previously in remission. Imaging findings that were deemed indicative of active or relapsing disease were new or persistent vessel wall thickening plus enhancement, worsening stenosis, or aneurysm.

Descriptive statistical analysis was performed using GraphPad Prism 6.0. Patients with SIE were compared to those without SIE using Fisher's exact test for categorical variables and unpaired $t$ test for continuous variables; $\mathrm{p}$ values $<0.05$ were considered statistically significant.

\section{RESULTS}

Patient demographics and frequency of SIE. This cohort included 52 patients: 50 meeting the ACR criteria for TA, and 2 based on physician opinion. Two patients were diagnosed with TA based on physician opinion: a 22-year-old female presenting with MI, elevated inflammatory markers, and aortic wall thickening on MRA, and a 47-year-old female with carotidynia, weight loss, and elevated erythrocyte sedimentation rate with subclavian stenosis and aortic wall thickening on imaging. Most were female (51/52, 98\%), and $42 \%$ were of European descent. The mean age at diagnosis was 31 years (SD 12) and the mean followup time was 6 years (SD 5). On vascular imaging, the most commonly affected arteries were the aorta, carotid, and subclavian arteries at diagnosis (Supplementary Table 1, available with the online version of this article). Of the 52 patients included in the study, 17 had followup imaging. New lesions were more frequently detected in the first 6 months of followup, and after 6 months, imaging was mostly stable. There were no significant differences in new imaging findings at followup between patients with and without SIE (Supplementary Table 2).

Within this population, 15/52 (29\%) had a single event over the course of the study. Most SIE (87\%) occurred at or before diagnosis. Of the patients with SIE, 5/15 patients (33\%) had CVA (2 presented with ischemic stroke and 2 presented with TIA at or before diagnosis; 1 presented with TIA at 6 months), 6/15 (40\%) had CV SIE (2 with STEMI, 2 with NSTEMI, and 1 with ischemic cardiomyopathy at or before diagnosis; 1 with STEMI at 6 months), and 4/15 $(27 \%)$ presented at or before diagnosis with severe limb ischemia. No patients had ischemia-induced blindness or severe ischemic bowel.

Characteristics of patients with and without SIE. Demographics and presenting features of the study population are summarized in Table 1 and Supplementary Table 1 (available with the online version of this article). The median diagnostic delay for patients with SIE was 5 months (range, 0-182) and for those without SIE, 13 months (range, $1-446 ; p=0.0313$ ). Patients with SIE presented more often with symptoms related to their specific type of SIE. For example, patients with CV SIE (ACS or ischemic cardiomyopathy) were more likely to have classic MI symptoms on presentation, such as angina and dyspnea; patients with severe limb ischemia were more likely to present with cutaneous manifestations, including livedo reticularis and digital ulcerations. These traditional CV disease (CVD) risk factors were infrequent at baseline: diabetes, hypertension (HTN), dyslipidemia (precise lipid levels were not available), smoking, obesity (body mass index $>30$ ), and family history of CVD. The study was not powered to determine whether traditional CVD risks were associated with SIE. HTN was present at baseline in 3 SIE patients and an additional 3 patients developed HTN over the followup period. Data on other CVD risk factors at followup were not collected.

Medical treatments and vascular interventions. At diagnosis, 13/15 (87\%) of SIE patients and 30/37 (81\%) of non-SIE patients began taking prednisone, at mean doses of 51.5 (SD 9.9) $\mathrm{mg} /$ day and 53.3 (17.0) $\mathrm{mg} /$ day, respectively ( $p>0.05$; Table 2). For patients diagnosed after 2008, the starting dose of prednisone was higher than those diagnosed before then: 47.3 (SD 25.3) versus 33.81 (20.8) $\mathrm{mg}$ /day $(\mathrm{p}=0.038)$. Patients with SIE were more likely than those

Personal non-commercial use only. The Journal of Rheumatology Copyright $@$ 2020 . All rights reserved. 
Table 1. Demographics and presentation of TA patients with and without SIE at diagnosis.

\begin{tabular}{|c|c|c|}
\hline Features & With SIE, $n=15$ & Without SIE, $\mathrm{n}=37$ \\
\hline Age, yrs, mean \pm SD & $31 \pm 12$ & $32 \pm 11$ \\
\hline Diagnostic delay, mos, mean $\pm \mathrm{SD}$ & D $27 \pm 58$ & $46 \pm 92$ \\
\hline \multicolumn{3}{|l|}{ Sex } \\
\hline Female & $15(100)$ & $36(97)$ \\
\hline \multicolumn{3}{|l|}{ Ethnicity } \\
\hline Asian & $1(7)$ & $4(11)$ \\
\hline Black & 0 & $3(8)$ \\
\hline European descent & $8(53)$ & $14(38)$ \\
\hline East Indian & $4(27)$ & $6(16)$ \\
\hline Hispanic & 0 & $3(8)$ \\
\hline Other & $2(13)$ & 7 (19) \\
\hline \multicolumn{3}{|l|}{ CVD risk factors } \\
\hline Diabetes & 0 & 0 \\
\hline Hypertension & $2(13)$ & $2(5)$ \\
\hline Dyslipidemia & $1(7)$ & 0 \\
\hline Prior or current smoking & $4(27)$ & $5(14)$ \\
\hline Obesity & 0 & 0 \\
\hline Family history & $1(7)$ & $4(11)$ \\
\hline \multicolumn{3}{|l|}{ Presenting symptoms* } \\
\hline Systemic & $10(67)$ & $32(87)$ \\
\hline Rheumatic & $6(40)$ & $17(46)$ \\
\hline Ocular & $3(20)$ & $5(14)$ \\
\hline Neurologic & $5(33)$ & $12(32)$ \\
\hline $\mathrm{CV}$ & $15(100)$ & $37(100)$ \\
\hline Cutaneous & $7(47)$ & $4(11)$ \\
\hline Gastrointestinal & $1(7)$ & $1(3)$ \\
\hline Genitourinary & 0 & $1(3)$ \\
\hline Respiratory & $5(33)$ & $4(11)$ \\
\hline \multicolumn{3}{|l|}{ Laboratory results, mean $\pm \mathrm{SD}$} \\
\hline Hemoglobin, g/l & $113.21 \pm 14.73$ & $122.08 \pm 19.76$ \\
\hline $\mathrm{ESR}, \mathrm{mm} / \mathrm{h}$ & $41.73 \pm 23.12$ & $42.26 \pm 21.33$ \\
\hline $\mathrm{CRP}, \mathrm{mg} / 1$ & $31.62 \pm 32.39$ & $33.28 \pm 28.00$ \\
\hline Creatinine, $\mu \mathrm{mol} / 1$ & $66.46 \pm 13.01$ & $63.42 \pm 18.20$ \\
\hline
\end{tabular}

Data are presented as numbers (\%) unless otherwise indicated. *Details of presenting symptoms provided in Supplementary Table 1 (available with the online version of this article). TA: Takayasu arteritis; SIE: severe ischemic event; CV: cardiovascular; CVD: CV disease; ESR: erythrocyte sedimentation rate; CRP: C-reactive protein.

without SIE to be placed on combination therapy [corticosteroids (CS) plus an immunosuppressant]: 8/15 (53\%) versus 9/37 (25\%), respectively $(\mathrm{p}=0.04$; Table 2$)$. SIE patients were also more likely to begin taking antiplatelet therapy: $14 / 15(93 \%)$ versus $19 / 37(51 \% ; \mathrm{p}=0.004)$.

The prednisone dosage decreased over time for both groups. Non-SIE patients were more likely to continue prednisone treatment than SIE patients $[19 / 35$ (59\%) vs $3 / 13(23 \%), p=0.01$; Table 2]. At last followup, SIE and non-SIE patients diagnosed after 2008 were more likely to be prescribed immunosuppressants combined with CS than those diagnosed before then: $11 / 20(55 \%)$ versus $5 / 15(33 \%$; $\mathrm{p}=0.014)$. There were no significant differences between SIE and non-SIE patients in the use of immunosuppressants, biologics, or antihypertensive drugs at the followup visits (Table 2, and Supplementary Table 3, available with the online version of this article). As per usual care at our centers and because of drug reimbursement practices, biologic therapies were used after failure of many conventional immunosuppressants combined with CS. Before 2008, only 1/15 patients $(7 \%)$ was treated with biologic drugs, compared to $5 / 20(25 \%)$ after $2008(\mathrm{p}>0.05)$.

Eleven revascularization procedures were performed on 8 patients with SIE and 2 without SIE $(\mathrm{p}=0.00007)$. The procedures were 3 coronary artery stents, 2 coronary artery bypass surgeries, 2 embolectomies in limb vessels, 1 subclavian artery angioplasty, 1 right subclavian artery, 1 renal, and 1 aortic stent.

Patient outcomes and disease activity. Table 3 summarizes the outcomes of SIE compared to non-SIE patients. There were no significant differences in relapses over the course of the study: 6/15 SIE patients relapsed (40\%), with 1 of them relapsing twice, versus $8 / 37(21 \%)$ in the non-SIE patients, with 2 of them relapsing twice $(\mathrm{p}=0.190)$. None of the patients died during the study period. There was no difference in the mean ITAS for SIE versus non-SIE patients at diagnosis: 11.4 (SD 5.8) versus 9.19 (SD 3.9). Despite patients diagnosed after 2008 being treated more aggressively, the outcomes were not different when compared to those diagnosed before 2008 .

\section{DISCUSSION}

In this cohort of Canadian patients with $\mathrm{TA}$ and long followup, SIE were common and occurred mostly at or before diagnosis. The most frequent SIE were ACS and CVA, occurring in $12 \%$ and $10 \%$, respectively.

The proportion of patients experiencing SIE in this cohort is consistent with prior studies of TA that estimated the prevalence of stroke or TIA to be $8-15 \% 4,13$, and of myocardial infarction, 3-22\% $\%^{4,6,14}$. Conversely, the observed rate of new SIE at followup was lower than reported in a recent metaanalysis, which estimated $9.2 \%$ and $7.4 \%$ of patients had a stroke or MI, respectively, at followup ${ }^{4}$. In our study with a mean followup of 6 years, only 2 patients $(1.3 \%)$ experienced a new SIE (1 TIA and $1 \mathrm{MI}$ ), occurring within the first 6 months after diagnosis. There are several possible explanations for this difference: our population included more patients of European descent, a demographic reported to have less severe disease ${ }^{15}$, and all of the SIE patients in our study received antiplatelet therapy at diagnosis, which has been shown to reduce ischemic events in an observational study of patients with $\mathrm{TA}^{16}$. The patients in our study were also started on aggressive treatment with CS combined with immunosuppressants at diagnosis.

Over the course of followup, patients with SIE had similar disease activity based on ITAS compared to non-SIE patients. Despite SIE patients having numerically more relapses, they were more likely to not be taking CS. The reason for this finding is unclear, but it is possible that physicians aimed to avoid CS exposure in patients with SIE

Personal non-commercial use only. The Journal of Rheumatology Copyright @ 2020 . All rights reserved. 
Table 2. Treatments in SIE and non-SIE patients.

\begin{tabular}{|c|c|c|c|}
\hline Medications & With SIE & Without SIE & $\mathrm{p}$ \\
\hline Medications started at diagnosis & $\mathrm{n}=15$ & $\mathrm{n}=37$ & \\
\hline Prednisone & $13(87)$ & $30(81)$ & 0.63 \\
\hline Mean prednisone dosage in $\mathrm{mg} / \mathrm{day} \pm \mathrm{SD}$ & $51.5 \pm 9.9$ & $53.3 \pm 17.0$ & 0.363 \\
\hline Corticosteroid + immunosuppressants & $8(53)$ & $9(25)$ & 0.04 \\
\hline Antiplatelet & $14(93)$ & $19(51)$ & 0.004 \\
\hline Anticoagulant & $2(13)$ & $3(8)$ & 0.56 \\
\hline Antihypertensive & $2(13)$ & $6(16)$ & 0.79 \\
\hline Medications at 6-month visit & $\mathrm{n}=15$ & $\mathrm{n}=36$ & \\
\hline Prednisone & $13(87)$ & $31(86)$ & 0.88 \\
\hline Mean prednisone dosage in $\mathrm{mg} / \mathrm{day} \pm \mathrm{SD}$ & $23.2 \pm 17.2$ & $23.1 \pm 15.3$ & 0.49 \\
\hline Corticosteroid + immunosuppressants & $10(67)$ & $21(58)$ & 0.56 \\
\hline Corticosteroid + biologics & 0 & $2(6)$ & 0.34 \\
\hline Any change in hypertensive medication & $2(13)$ & $3(8)$ & 0.57 \\
\hline Medications at 12 -month visit & $\mathrm{n}=13$ & $\mathrm{n}=35$ & \\
\hline Prednisone & $12(92)$ & $28(80)$ & 0.23 \\
\hline Mean prednisone dosage $\mathrm{mg} /$ day $\pm \mathrm{SD}$ & $8.6 \pm 4.8$ & $13.8 \pm 14.4$ & 0.11 \\
\hline Corticosteroid + immunosuppressants & $12(92)$ & $25(71)$ & 0.09 \\
\hline Corticosteroid + biologics & 0 & $3(9)$ & 0.21 \\
\hline Any change in hypertensive medication & $2(15)$ & $2(6)$ & 0.21 \\
\hline Medications at last followup* & $\mathrm{n}=13$ & $\mathrm{n}=35$ & \\
\hline Prednisone & $3(23)$ & $19(54)$ & 0.01 \\
\hline Mean prednisone dosage in $\mathrm{mg} / \mathrm{day} \pm \mathrm{SD}$ & $1.5 \pm 3.1$ & $5.0 \pm 8.2$ & 0.07 \\
\hline Corticosteroid + immunosuppressants & $8(61)$ & $15(43)$ & 0.07 \\
\hline Corticosteroid + biologics & $1(0.07)$ & $5(14)$ & 0.13 \\
\hline Any change in hypertensive medication & $2(15)$ & $2(6)$ & 0.06 \\
\hline
\end{tabular}

Table 3. Outcomes and disease activity scores in SIE and non-SIE patients.

\begin{tabular}{lccc}
\hline Outcomes & With SIE & Without SIE & $\mathrm{p}$ \\
\hline Outcomes at diagnosis & $\mathrm{n}=15$ & $\mathrm{n}=37$ & \\
Active & $15(100)$ & $37(100)$ & 1.00 \\
ITAS, mean \pm SD & $11.4 \pm 5.8$ & $9.19 \pm 3.9$ & 0.06 \\
Outcomes at 6-month visit & $\mathrm{n}=15$ & $\mathrm{n}=36$ & \\
Total remission/improving & $10(67)$ & $30(83)$ & 0.18 \\
Active & $1(6)$ & $2(6)$ & 0.84 \\
Relapse & $4(27)$ & $4(11)$ & 0.16 \\
ITAS, mean \pm SD & $1.27 \pm 1.7$ & $0.83 \pm 1.4$ & 0.17 \\
Outcomes at 12-month visit & $\mathrm{n}=13$ & $\mathrm{n}=35$ & \\
Total remission/improving & $12(92)$ & $28(80)$ & 0.23 \\
Active & 0 & $2(6)$ & 0.284 \\
Relapse & $1(8)$ & $5(14)$ & 0.40 \\
ITAS, mean \pm SD & $0.46 \pm 0.9$ & $0.71 \pm 1.6$ & 0.30 \\
Outcomes at last followup* & $\mathrm{n}=13$ & $\mathrm{n}=35$ & \\
Total remission/improving & $11(85)$ & $34(97)$ & $\mathbf{0 . 0 4}$ \\
Active & 0 & 0 & $\mathrm{~N} / \mathrm{A}$ \\
Relapse & $2(18)$ & $1(3)$ & $\mathbf{0 . 0 4}$ \\
ITAS, mean \pm SD & $0.08 \pm 0.28$ & $0.54 \pm 2.0$ & 0.19 \\
\hline
\end{tabular}

*Includes patients with last followup at $>12$ months from baseline. Data are presented as numbers (\%) unless otherwise indicated. Values in bold face are statistically significant. SIE: severe ischemic event; ITAS: Indian Takayasu Activity Score; N/A: not applicable.

because it is known to increase the risk of atherosclerotic events. The use of other immunosuppressants was similar for SIE and non-SIE patients.
Although detailed information on the management of all $\mathrm{CV}$ risk factors in this cohort was incomplete, the proportion of patients with traditional CV risk factors was low, as expected from a study population consisting predominantly of young women. Hence, mechanisms other than atherosclerosis are likely contributing to SIE in TA. For example, direct vascular inflammation from TA leading to stenosis, thrombosis, or embolism can directly cause ACS and CVA. Unfortunately, the imaging modalities used in this study (conventional angiogram, CTA, and 1.5T MRA) are limited in their ability to differentiate vascular inflammation from atherosclerosis ${ }^{17}$. Higher resolution MRA, cardiac MRI, and PET scan were not readily available at our centers.

Our study has some limitations, including its small sample size; thus, it was not possible to identify independent risk factors for SIE. We included all patients with TA seen at 3 large centers in Ontario, the most populous province of Canada, and the study sample reflects the rarity of TA in Canada. Data collection was retrospective, but patients lost to followup and missing data were minimal. In this real-world study, some important variables that are not routinely collected in usual care were not available, such as functional scores and quality- of-life indicators.

SIE were common in this cohort of patients with TA. Given that most SIE occurred at or before diagnosis, methods to diagnose TA and initiate treatments earlier may help prevent or minimize some of these complications.

Personal non-commercial use only. The Journal of Rheumatology Copyright () 2020. All rights reserved. 


\section{ONLINE SUPPLEMENT}

Supplementary material accompanies the online version of this article.

\section{REFERENCES}

1. Subramanyan R, Joy J, Balakrishnan KG. Natural history of aortoarteritis (Takayasu's disease). Circulation 1989;80:429-37.

2. Kim ES, Beckman J. Takayasu arteritis: challenges in diagnosis and management. Heart 2018;104:558-65.

3. Mason JC. Takayasu arteritis - advances in diagnosis and management. Nat Rev Rheumatol 2010;6:406-15.

4. Kim H, Barra L. Ischemic complications in Takayasu's arteritis: a meta-analysis. Semin Arthritis Rheum 2018;47:900-6.

5. de Paula LE, Alverne AR, Shinjo SK. Clinical and vascular features of Takayasu arteritis at the time of ischemic stroke. Acta Rheumatol Port 2013;38:248-51.

6. Comarmond C, Cluzel P, Toledano D, Costedoat-Chalumeau N, Isnard R, Gaudric J, et al. Findings of cardiac magnetic resonance imaging in asymptomatic myocardial ischemic disease in Takayasu arteritis. Am J Cardiol 2014;113:881-7.

7. Ashjazadeh N, Shokouhyar S, Ostovan MA. Takayasu arteritis presenting as sudden onset vision loss simulates multiple sclerosis: a case report. J Res Med Sci 2011;16 Suppl 1:S442-6.

8. Vanoli M, Daina E, Salvarani C, Sabbadini MG, Rossi C, Bacchiani G, et al; Itaka Study Group. Takayasu arteritis: a study of 104 Italian patients. Arthritis Rheum 2005;53:100-7.

9. Nunes C, Capela C, Pinto L, Assuncao A, Ferreira AM. Severe Takayasu arteritis complicated by mesenteric ischemia. J Med Cases 2017;8:383-7.
10. Aeschlimann FA, Barra L, Alsolaimani R, Benseler SM, Hebert D, Khalidi N, et al. Presentation and disease course of childhood-onset versus adult-onset Takayasu arteritis. Arthritis Rheumatol 2019;71:315-23.

11. Arend WP, Michel BA, Bloch DA, Hunder GG, Calabrese LH, Edworthy SM, et al. The American College of Rheumatology 1990 criteria for the classification of Takayasu arteritis. Arthritis Rheum 1990;33:1129-34.

12. Misra R, Danda D, Rajappa SM, Ghosh A, Gupta R, Mahendranath $\mathrm{KM}$, et al; Indian Rheumatology Vasculitis (IRAVAS) group. Development and initial validation of the Indian Takayasu Clinical Activity Score (ITAS2010). Rheumatology 2013;52:1795-801.

13. Duarte MM, Geraldes R, Sousa R, Alarcão J, Costa J. Stroke and transient ischemic attack in Takayasu's arteritis: a systematic review and meta-analysis. J Stroke Cerebrovasc Dis 2016;25:781-91.

14. Cavalli G, Tomelleri A, Di Napoli D, Baldissera E, Dagna L. Prevalence of Takayasu arteritis in young women with acute ischemic heart disease. Int J Cardiol 2018;252:21-3.

15. Arnaud L, Haroche J, Limal N, Toledano D, Gambotti L, Costedoat-Chalumeau N, et al. Takayasu arteritis in France: a single center retrospective study of 82 cases comparing white, North African, and black patients. Medicine 2010;89:1-17.

16. de Souza AW, Machado NP, Pereira VM, Arraes AE, Reis Neto ET, Mariz HA, et al. Antiplatelet therapy for the prevention of arterial ischemic events in Takayasu arteritis. Circ J 2010;74:1236-41.

17. Akazawa H, Ikeda U, Yamamoto K, Kuroda T, Shimada K. Hypercoagulable state in patients with Takayasu's arteritis. Thromb Haemost 1996;75:712-6. 